\title{
Prognostic Value of Circulating Regulatory T Cells for Worsening Heart Failure in Heart Failure Patients With Reduced Ejection Fraction
}

\author{
Naoko Окамото, ${ }^{1}$ MD, Takahisa Noma, ${ }^{1}$ MD, Yasuhiro Ishinara, ${ }^{1}$ MD, Yuka MiYauchi, ${ }^{1}$ MD, \\ Wataru Takabatake, ${ }^{1} \mathrm{MD}$, Souichi Oomizu, ${ }^{2} \mathrm{PhD}$, Genji Yamaoka, ${ }^{3} \mathrm{PhD}$, Makoto Ishizawa, ${ }^{1} \mathrm{MD}$, \\ Tsunetatsu Namba, ${ }^{1}$ MD, Kazushi MuraKami,,${ }^{1}$ MD, Yasuyoshi Iwado, ${ }^{1}$ MD, \\ Koji OHMori, ${ }^{1} \mathrm{MD}$, and Masakazu KoHno, ${ }^{1} \mathrm{MD}$
}

\begin{abstract}
SUMMARY
Regulatory T cells (Tregs) play a crucial role in the negative regulation of immune responses. Recent studies suggest that Tregs are involved in the pathogenesis of atherosclerosis and myocarditis. Here, we investigated the involvement of Tregs on worsening heart failure (HF) in patients with reduced ejection fraction (HF-REF). The study population consisted of $32 \mathrm{HF}-\mathrm{REF}$ patients who were hospitalized for worsening HF, and 18 control subjects. Cardiac function was evaluated by echocardiography. A single venous blood sample was collected before discharge. Circulating $\mathrm{T}$ cells were evaluated by flow cytometry. Tregs were defined as $\mathrm{CD}^{+}{ }^{+} \mathrm{CD} 25^{+} \mathrm{Foxp} 3^{+} \mathrm{T}$ cells, and the correlations between the frequency of Tregs and CRP, IL-6 and several echoparameters were analysed. Furthermore, all HF-REF patients were followed up to 12 months from discharge to examine the predictors of recurrent hospitalization.

In HF-REF patients, Tregs were significantly decreased $(5.9 \pm 1.4$ versus $8.0 \pm 2.2 \%, P<0.01)$, while $\mathrm{CD}^{+}{ }^{+} \mathrm{HLADR}^{+} \mathrm{T}$ cells were increased $(10.1 \pm 5.4$ versus $7.3 \pm 3.1 \%, P<0.05)$, compared with controls. Tregs were negatively correlated with left ventricular end-diastolic dimension, and levels of CRP and IL-6. Eleven of 32 HF-REF patients were rehospitalized for worsening HF within 12 months. Multivariate Cox regression analysis showed that CD4/ CD8 and frequency of Tregs were independent predictors for recurrent hospitalization. Furthermore, HF-REF patients expressing under $6 \% \mathrm{Treg} / \mathrm{CD} 4^{+} \mathrm{T}$ cells showed a significantly higher incidence of recurrent hospitalization for worsening HF within 12 months.

Our data suggest that Tregs might be involved in the pathogenesis of decompensated HF, and may be a novel predictor of poor prognosis in HF-REF patients. (Int Heart J 2014; 55: 271-277)
\end{abstract}

Key words: Inflammation

$\mathrm{O}$ ptimal medical therapy for chronic heart failure (HF) patients has clearly improved mortality rates. However, despite treatment advances, the frequency of recurrent hospitalization due to worsening heart failure remains high, which is considered to cause further deterioration of cardiac function and reduced quality of life. ${ }^{1,2)}$ Clinical, electrocardiographic, echocardiographic, and biochemical markers of adverse prognosis have been used in an attempt to identify patients at high risk for developing life-threatening cardiac events, and to determine whether patients may benefit from a more intensive pharmacological and/or interventional strategy. ${ }^{3)}$

Activation of the immune system and inflammation in HF patients is considered to play an important role in the progression of heart failure. ${ }^{4)}$ In particular, variations in leukocyte differentials, including lymphocyte, monocyte, eosinophil and mast cells, have identified a high-risk subset of HF patients. A low lymphocyte count is a predictor of poor outcome in either chronic or hospitalized HF patients, despite widely heterogeneous study designs. ${ }^{5)}$ Furthermore, circulating T cells, but not monocytes, are markedly activated in HF patients as assessed by both enhanced mRNA levels of several inflammatory cytokines and by increased surface expression of activation markers.)

$\mathrm{CD} 4^{+} \mathrm{CD} 25^{+}$regulatory $\mathrm{T}$ cells (Tregs) were first described by Sakaguchi, et al, ${ }^{7}$ and were found to specifically express the gene for forkhead/winged helix transcription factor (Foxp3). ${ }^{8}$ Tregs are now known to play a critical role in maintaining peripheral tolerance and controlling immune responses, where they typically control the behaviour of other T cell populations, although they can also influence innate immune system activity. A decrease or functional abnormality in Tregs can

From the Departments of ${ }^{1}$ Cardiorenal and Cerebrovascular Medicine, ${ }^{2}$ Immunology, and ${ }^{3}$ Clinical Laboratory, Faculty of Medicine, Kagawa University, Kagawa, Japan.

Address for correspondence: Takahisa Noma, MD, Department of Cardiorenal and Cerebrovascular Medicine, Faculty of Medicine, Kagawa University, 1750-1 Ikenobe, Miki-cho, Kita-gun, Kagawa 761-0793, Japan. E-mail: noma@med.kagawa-u.ac.jp

Received for publication November 14, 2013. Revised and accepted December 14, 2013.

Released advance online J-STAGE May 12, 2014.

All rights are reserved to the International Heart Journal Association. 
result in various autoimmune diseases. ${ }^{9-11)}$ Regarding heart failure, Tang, et al reported an association between peripheral Tregs and heart failure in HF patients who were stable on optimal therapy for at least 3 months. ${ }^{12)}$ Furthermore, Kvakan, et al reported that the transfer of Tregs ameliorated angiotensin II induced cardiac damage and improved electric remodelling in mice. ${ }^{13)}$ Matsumoto, et al also reported that transfer of Tregs attenuated myocardial infarction-induced ventricular remodelling in mice. ${ }^{14)}$ These findings suggest that peripheral circulating Tregs might be involved in the pathogenesis of left ventricular dysfunction during development of heart failure.

In the present study, we hypothesized that a decrease in circulating Tregs may be associated with activation of the immune system and pathogenesis of failing myocardium, which might result in frequent recurrent hospitalization for worsening $\mathrm{HF}$ in patients with reduced ejection fraction (HF-REF).

\section{Methods}

Patients: A total of 50 patients were enrolled in this study. The study population consisted of 32 chronic HF patients with reduced ejection fraction less than $50 \%$ who required hospitalization because of worsening HF. Worsening HF patients were defined as those with dyspnea, pulmonary congestion in chest $\mathrm{X}$-ray, and who required intravenous treatment at hospitalization. The etiologies of HF were ischemic cardiomyopathy $(n=$ $8)$, nonischemic cardiomyopathy $(n=18)$, or valvular heart disease $(n=6)$. Diagnosis of etiology of HF was based on clinical history, echocardiography, chest X-rays, electrocardiography, and cardiac catheterization. Control subjects were 18 non-HF patients with an ejection fraction greater than $60 \%$, and who were admitted at Kagawa University Hospital for various causes including angina pectoris and arrhythmia. The baseline characteristics of the subjects in this study are shown in Table I. Exclusion criteria were history of malignant disease, chronic inflammatory disease, connective tissue disease, hemodialysis, acute myocardial infarction, and/or acute myocarditis.

A single venous blood sample was collected before discharge when subjects exhibited no symptoms of inflammation, including fever and increased white blood cells, to avoid the influence of infection for measurement of soluble mediators, determination of cellular differentials and expression of cell surface molecules. All patients were followed up to 12 months from discharge to evaluate recurrent hospitalization for worsening HF. This study was approved by the Committee of Ethics at Kagawa University, and all patients provided written informed consent.

Three-colour flow cytometric analysis of white blood cells: Monoclonal antibodies for flow cytometric analysis included fluorescein isothiocyanate (FITC)- and phycoerythrin (PE)conjugated anti-human CD25 monoclonal antibodies (mAbs) (Dako, Tokyo). FITC-conjugated anti-human CD28 and iso-

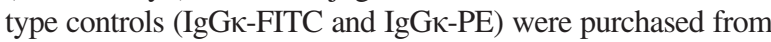
BD Bioscience (San Diego, CA, USA). Phycoerythrincyanin 5.1 (PC5)-FITC and -PE-conjugated anti-human CD45, CD4 and HLADR were purchased from Beckman Coulter (Miami, FL, USA). PE-conjugated rat IgG2aK was purchased from Beckman Dickinson (Franklin Lakes, NJ, USA). Whole blood samples obtained from all patients were analysed by three-col-
Table I. Basal Clinical Characteristics in HF-REF Patients Hospitalized for Worsening HF

\begin{tabular}{|c|c|c|}
\hline & $\begin{array}{c}\text { Control } \\
n=18\end{array}$ & $\begin{array}{c}\mathrm{HF} \\
n=32\end{array}$ \\
\hline Age, years & $65.4 \pm 3.3$ & $67.7 \pm 2.2$ \\
\hline Sex (male/female (\%)) & $13 / 5(72.2 / 27.8)$ & $24 / 8(75 / 25)$ \\
\hline BMI, $\mathrm{kg} / \mathrm{m}^{2}$ & $26.0 \pm 0.8$ & $23.0 \pm 0.8^{*}$ \\
\hline Diabetes mellitus (\%) & $4(22.2)$ & $11(34.4)$ \\
\hline Hypertension (\%) & $12(66.7)$ & $14(43.8)$ \\
\hline Dyslipidemia (\%) & $12(66.7)$ & $14(43.8)$ \\
\hline Chronic kidney disease & $7(38.9)$ & $20(62.5)$ \\
\hline \multicolumn{3}{|l|}{ NYHA (\%) } \\
\hline $\mathrm{II} / \mathrm{III}$ & & $19(59.4)$ \\
\hline IV & & $13(40.6)$ \\
\hline \multicolumn{3}{|l|}{ Etiology (\%) } \\
\hline ICM & & $8(25.0)$ \\
\hline DCM (NICM) & & $18(56.3)$ \\
\hline Valve & & $6(18.8)$ \\
\hline \multicolumn{3}{|l|}{ Echoparameter } \\
\hline $\mathrm{EF}, \%$ & $64.7 \pm 1.9$ & $33.3 \pm 1.7^{* *}$ \\
\hline LVDd, mm & $48.4 \pm 1.3$ & $62.0 \pm 1.7^{* *}$ \\
\hline LVDs, mm & $30.3 \pm 1.5$ & $52.7 \pm 2.3^{* *}$ \\
\hline \multicolumn{3}{|l|}{ Medication, \% } \\
\hline Diuretics & 16.7 & $96.9^{* *}$ \\
\hline ACEI/ARB & 61.1 & 81.3 \\
\hline Digitalis & 0.0 & $21.9^{*}$ \\
\hline$\beta$ blocker & 33.3 & $93.8^{* *}$ \\
\hline statin & 66.7 & 37.5 \\
\hline $\mathrm{WBC}, / \mathrm{uL}$ & $6012.4 \pm 236.8$ & $5692.5 \pm 333.6$ \\
\hline Lynphocyte, /uL & $1862.6 \pm 148.7$ & $1617.3 \pm 135.7$ \\
\hline $\mathrm{Hb}, \mathrm{g} / \mathrm{dL}$ & $14.1 \pm 0.4$ & $13.2 \pm 0.3$ \\
\hline Cre, mg/dL & $0.8 \pm 0.0$ & $1.1 \pm 0.1^{* *}$ \\
\hline $\mathrm{eGFR}, \mathrm{mL} / \mathrm{mim} / 1.73 \mathrm{~m}^{2}$ & $72.4 \pm 4.4$ & $56.5 \pm 4.4^{*}$ \\
\hline $\mathrm{HbAlc}, \%$ & $5.8 \pm 0.2$ & $5.9 \pm 0.2$ \\
\hline \multicolumn{3}{|l|}{ Biomarkers } \\
\hline NT-proBNP, pg/mL & $177.9 \pm 76.4$ & $3017.1 \pm 982.7^{* *}$ \\
\hline hs-CRP, pg/mL & $650.8 \pm 225.5$ & $1595.9 \pm 307.4^{*}$ \\
\hline $\mathrm{IL}-6, \mathrm{pg} / \mathrm{mL}$ & $2.3 \pm 0.4$ & $6.7 \pm 1.7^{*}$ \\
\hline Troponin I, ng/mL & $0.003 \pm 0.002$ & $0.024 \pm 0.007^{*}$ \\
\hline
\end{tabular}

our flow cytometry. In brief, whole blood cells were incubated with PC5-, PE- and FITC-conjugated mAbs for 15 minutes at room temperature. The erythrocytes in each sample were haemolysed and then washed with phosphate buffered saline (PBS). Finally, the stained cells were analysed with CYTOMICS FC 500 (Beckman Coulter). All T cell subsets by surface stain were expressed as percentage of total $\mathrm{CD} 45^{+} \mathrm{T}$ cells. Representative blots are shown in Figure 1 (A, B, E, F).

Circulating blood mononuclear cell (PBMC) isolation: Whole blood samples were collected from all patients in both the HF and control groups, and PBMCs were isolated by FicollPaque $^{\mathrm{TM}}$ PLUS density gradient centrifugation (GE Healthcare, Buckinghamshire, England) at $2100 \mathrm{rpm}$ for 10 minutes at $4^{\circ} \mathrm{C}$ according to the manufacturer's protocol.

Three-colour flow cytometric analysis of Tregs: PBMCs were isolated as described above. Cells were aliquoted into tubes and washed once in PBS, and then resuspended to a density of $10^{6}$ cells/mL. PE- anti-human Foxp3 was purchased from eBioscience (San Diego, CA, USA). For Tregs analysis, cells were incubated with PC5-anti-human CD4, FITC-anti-human CD25, and FITC-anti-human HLADR. After surface staining, the cells were fixed, permeabilized and stained with PE-antiFoxp3 mAb according to the manufacturer's protocol. Isotype 
$<$ HF-REF>
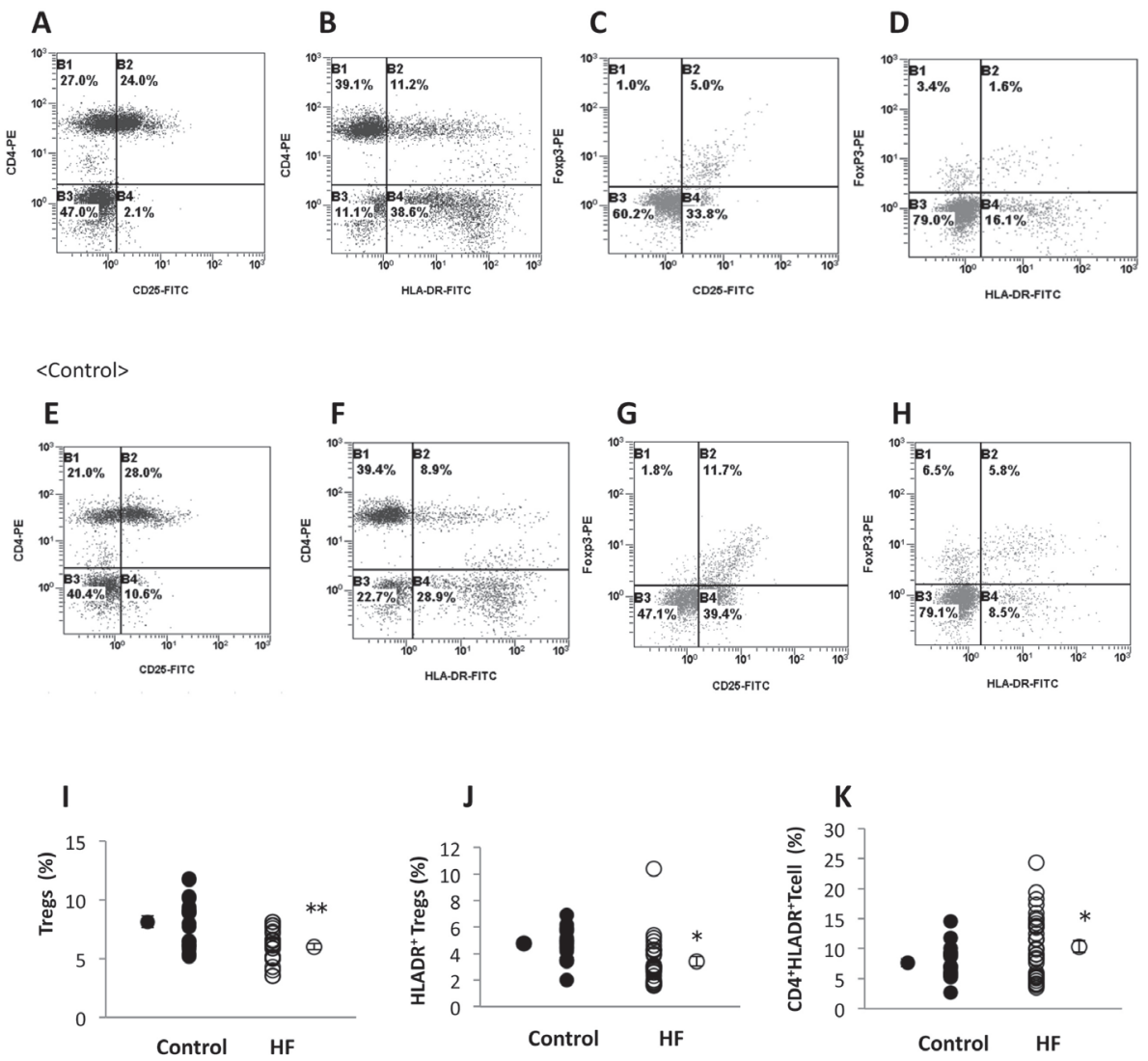

Figure 1. Representative flow cytometric profiles of circulating blood lymphocytes in control and HF patients and comparison of T cell subsets between HF patients and control subjects by FCM. FCM pictures of one person from the HF group (A-D) and the control group $(\mathbf{E}-\mathbf{H})$. (A, B, E, F) Surface stain and gated on CD45. (C, D, G, H) Intracellular stain and gated on CD4. Percentage of Tregs was significantly decreased in HF patients (I). Percentage of active Tregs (HLADR is an activation marker) was increased in HF patients ( $\mathbf{J}$ ). Percentage of active CD4 ${ }^{+} \mathrm{T}$ cells was significantly increased in HF patients $(\mathbf{K})$. Values are the mean \pm SEM. ${ }^{*} P<0.05,{ }^{* *} P<0.01$ versus control subjects by Student's $t$-test.

controls were given to enable correct compensation and confirm antibody specificity. Stained cells were analysed with CYTOMICS FC 500 (Beckman Coulter). All T cell subsets by intracellular stain were expressed as a percentage of total $\mathrm{CD} 4^{+} \mathrm{T}$ cells. Representative blots are shown in Figure 1 (C, D, $\mathrm{G}, \mathrm{H})$.

Measurement of plasma biomarkers: After centrifugation, serum samples were frozen and stored at $-80^{\circ} \mathrm{C}$ until use. High sensitivity C-reactive protein (hs-CRP) was assessed by immunonephelometry (Siemens Healthcare Diagnostic Inc., Tokyo). The N-terminal fragment of pro-brain natriuretic peptide (NTproBNP) was measured by electrochemiluminescence immunoassay (Roche Diagnostics, Tokyo). High sensitivity troponin I (hs-TnI) was measured by chemiluminescence immunoassay (Siemens Healthcare Diagnostic Inc.). IL-6 was measured by chemiluminescence enzyme immunoassay (Fujirebio, Tokyo). TNF- $\alpha$, IL- $1 \beta$, and IL-10 were measured by Quantikine ELI$\mathrm{SA}^{\circledR}$ according to the manufacturer's protocol (R\&D Systems, Inc.).

Statistical analysis: Comparisons of HF patients versus control subjects were performed using the two tailed Student $t$-test where distributions of data were normal, and continuous val- ues are expressed as the mean \pm SEM. Categorical variables are expressed as number and percentage, and the chi-square test was used to compare categorical variables. Coefficients of correlation $(r)$ were calculated using Pearson's correlation coefficient. Univariate Cox regression analysis was used to identify the cofactors with significant effects on rehospitalization. A step-wise multivariate Cox regression model was used to determine the independent prognostic factors for recurrent hospitalization.

Recurrent hospitalization within 12 months from discharge was estimated using the Kaplan-Meier survival curve and log rank test to assess the significance of differences between Treg-low and Treg-high. $P$-values of $<0.05$ were considered statistically significant. Statistical analysis of the results was performed using the IBM SPSS Statistics version 21.0.

\section{RESULTS}

Clinical characteristics in HF-REF patients hospitalized for worsening HF: The baseline clinical characteristics of the study subjects are detailed in Table I. In HF-REF patients hos- 
pitalized for worsening HF, $56.3 \%$ of patients were diagnosed as dilated cardiomyopathy, with a mean $\mathrm{EF}$ of $33.3 \pm 1.7 \%$ and a left ventricular end-diastolic dimension of $62.0 \pm 1.7 \mathrm{~mm}$. NYHA class IV was observed in $40.6 \%$ of the patients at the time of admission. An angiotensin converting enzyme inhibitor (ACEI)/angiotensin II receptor blocker (ARB) was being taken by $81.3 \%$ of the patients and $93.8 \%$ of the patients were taking a beta-blocker. BMI was significantly lower in the HF patients than in the control subjects.

Peripheral blood biomarkers in the HF-REF patients and control subjects are also shown in Table I. There were no significant differences in white blood cell count and lymphocyte count, and hemoglobin. A decrease in eGFR and an increase in NT-proBNP were observed in HF-REF patients. Neither of the inflammatory cytokines IL-6 or hs-CRP was significantly elevated in HF-REF patients. The levels of hs-TnI, a marker of cardiac injury, were also not significantly elevated. There were no significant differences in IL- $1 \beta$ and IL-10 between the HFREF patients and control subjects $(1.36 \pm 2.11$ versus $2.21 \pm$
$2.64 \mathrm{pg} / \mathrm{mL}, 12.95 \pm 21.86$ versus $16.93 \pm 23.00 \mathrm{pg} / \mathrm{mL}$, respectively). TNF- $\alpha$ was undetectable in both HF-REF patients and control subjects.

Decrease in circulating Tregs and HLADR-positive Tregs in HFREF patients hospitalized for worsening HF: Representative flow cytometric profiles of circulating blood lymphocytes in HF-REF patients and control subjects are shown in Figure 1. There were no significant differences in the CD4/CD8 ratio $(1.77 \pm 0.18$ versus $1.94 \pm 0.23, P=\mathrm{NS}), \mathrm{CD}^{+} \mathrm{CD} 25^{+}(16.5 \pm$ 5.8 versus $18.5 \pm 7.1 \%, P=\mathrm{NS}), \mathrm{CD}_{25}{ }^{+} \mathrm{HLADR}^{+}(7.6 \pm 5.9$ versus $6.3 \pm 2.3 \%, P=\mathrm{NS})$ and $\mathrm{CD} 25^{+} \mathrm{CD} 28^{+}(50.3 \pm 13.1$ versus $54.0 \pm 13.9 \%, P=\mathrm{NS}$ ) between HF-REF patients and control subjects. CD $4^{+}$Foxp $3^{+}$T cells (Tregs) were significantly decreased in HF-REF patients (Figure 1I). Furthermore, $\mathrm{HLADR}^{+}$Tregs, which is an active Treg, was significantly decreased in HF-REF patients (Figure $1 \mathrm{~J}$ ). Active $\mathrm{CD}^{+}{ }^{+} \mathrm{T}$ cells, represented as $\mathrm{CD}^{+}{ }^{+} \mathrm{HLADR}^{+} \mathrm{T}$ cells, were significantly increased in HF-REF patients (Figure 1K). These data indicated that a decrease in Tregs might be involved in the sustained in-
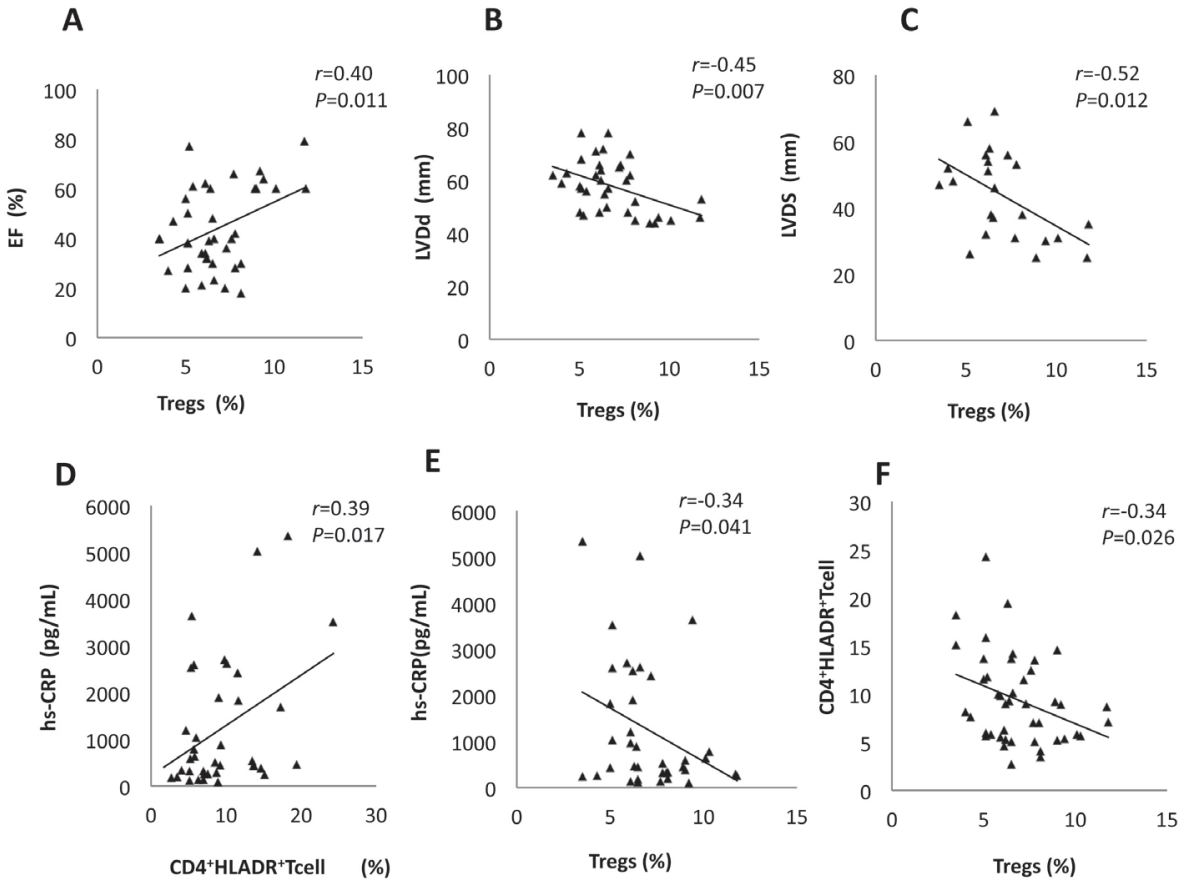

G

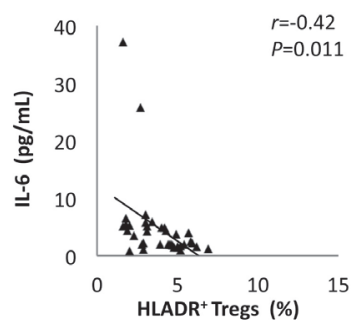

Figure 2. Correlation between Tregs and echoparameters and inflammation and IL-6. (A, B, C) The echoparameters of left ventricular ejection fraction (E, F) and left ventricular dimension diastolic/systolic (LVDd/s) were correlated with percentage of Tregs. (D, E) Highly sensitive CRP was correlated with negative percentage of Tregs and positively active $\mathrm{CD} 4^{+} \mathrm{T}$ cells. (F) Tregs were negatively correlated with active $\mathrm{CD} 4^{+} \mathrm{T}$ cells. $(\mathbf{G})$ Active Tregs were negatively correlated with IL-6. Coefficients of correlation $(r)$ were calculated by the Pearson's correlation coefficient. 
flammation in HF-REF patients hospitalized for worsening HF. Correlation of Tregs with cardiac function and inflammatory cytokines: Next, we examined the correlation of Tregs and $\mathrm{CD}^{+}{ }^{+} \mathrm{HLADR}^{+} \mathrm{T}$ cells with cardiac function evaluated by several echoparameters and the level of inflammatory cytokines. As shown in Figure 2, Tregs were positively correlated with $\mathrm{EF}$, but negatively correlated with left ventricular end diastolic and systolic dimension (Figure 2A, B, C). hs-CRP was positively correlated with $\mathrm{CD}^{+} \mathrm{HLADR}^{+} \mathrm{T}$ cells (Figure 2D), which was significantly higher in HF-REF patients compared with controls (Figure $1 \mathrm{~K}$ ), indicating that active $\mathrm{CD} 4^{+} \mathrm{T}$ cells may be involved with inflammation. By contrast, there was a negative correlation between hs-CRP and Tregs (Figure 2E). Furthermore, Tregs were negatively correlated with CD $4^{+} \mathrm{HLADR}^{+} \mathrm{T}$ cells (Figure $2 \mathrm{~F}$ ). IL-6 was also negatively correlated with $\mathrm{CD} 4^{+}$Foxp $3^{+} \mathrm{HLADR}^{+} \mathrm{T}$ cells (Figure $2 \mathrm{G}$ ). These findings suggest that circulating Tregs may be associated with inflammation and left ventricular dysfunction in HFREF patients.

Decrease in circulating Tregs is an independent predictor for recurrent hospitalization within 12 months in HF-REF patients: In this study, 11 of $32 \mathrm{HF}-\mathrm{REF}$ patients were rehospitalized for worsening HF within 12 months from discharge. In the univariate Cox regression model, the CD4/CD8 ratio, and percentages of $\mathrm{CD}^{+} \mathrm{CD} 25^{+}, \mathrm{CD} 25^{+} \mathrm{HLADR}^{+}$, Tregs, and $\mathrm{HLADR}^{+}$Tregs were associated with recurrent hospitalization in HF-REF patients (Table II). A step-wise multivariate Cox regression analysis showed that CD4/CD8 and percentage of Tregs were independent predictors for recurrent hospitalization in HF-REF patients (Table III). To determine the predictive value of percentage of Tregs on recurrent hospitalization, we divided the HF-REF patients according to the median percentage of Tregs, which was $6 \%$. Kaplan-Meier curves and log-rank testing revealed that HF-REF patients with a high percentage of Tregs (over $6 \%$ of total $\mathrm{CD}^{+} \mathrm{T}$ cells) had a significantly lower incidence of recurrent hospitalization within 12 months from discharge (Figure 3 ).

\section{DiscuSSION}

The present study demonstrates that the decrease in circulating Tregs is associated with inflammation and left ventricular dysfunction in HF-REF patients hospitalized for worsening HF. Further, the lower frequency of Tregs is an independent predictor of recurrent hospitalization for worsening HF, rather than LVEF, LV dimension or NT-proBNP. These findings indicate that the circulating Tregs may play a role at least in the development of $\mathrm{HF}$, independent of cardiac function and volume status. In the present study, we found a marked decrease in Tregs in HF-REF patients who were hospitalized for worsening HF compared with the frequency of Tregs in chronic heart failure patients previously reported. Furthermore, much lower frequencies of Tregs were observed in 'unstable' patients who were hospitalized more than twice within 12 months.

It is known that systemic inflammation plays an important role in the development and progression of heart failure. Although the direct role of Tregs on inflammation remains to be clarified, there are several reports demonstrating multiple suppressive mechanisms. Wing, et al reported that Tregs constitutively express the surface molecule cytotoxic T lym-
Table II. Univariate Predictors of Recurrent Hospitalization for Worsening $\mathrm{HF}$

\begin{tabular}{|c|c|c|c|c|}
\hline \multirow[b]{2}{*}{ Age, years } & \multirow{2}{*}{$\frac{P}{0.845}$} & \multirow{2}{*}{$\frac{\text { Hazard ratio }}{1.005}$} & \multicolumn{2}{|c|}{$95 \%$ CI } \\
\hline & & & 0.958 & 1.053 \\
\hline CD4/CD8 & 0.009 & 1.965 & 1.187 & 3.253 \\
\hline $\mathrm{CD} 4^{+} \mathrm{CD} 25^{+}, \%$ & 0.037 & 1.120 & 1.007 & 1.246 \\
\hline $\mathrm{CD}^{+} \mathrm{HLADR}^{+}, \%$ & 0.261 & 0.932 & 0.824 & 1.054 \\
\hline $\mathrm{CD}_{25}{ }^{+} \mathrm{HLADR}^{+}, \%$ & 0.009 & 0.671 & 0.498 & 0.905 \\
\hline $\mathrm{CD} 25^{+} \mathrm{CD} 28^{+}, \%$ & 0.920 & 1.002 & 0.955 & 1.052 \\
\hline Tregs, \% & 0.018 & 0.565 & 0.352 & 0.908 \\
\hline HLADR ${ }^{+}$Tregs, \% & 0.008 & 0.272 & 0.103 & 0.716 \\
\hline NT-proBNP, pg/mL & 0.858 & 1.000 & 1.000 & 1.000 \\
\hline Hs-CRP, pg/mL & 0.904 & 1.000 & 0.999 & 1.000 \\
\hline $\mathrm{IL}-6, \mathrm{pg} / \mathrm{mL}$ & 0.418 & 1.028 & 0.961 & 1.100 \\
\hline Troponin I, pg/mL & 0.606 & 0.989 & 0.948 & 1.032 \\
\hline \multicolumn{5}{|l|}{ Echoparameters } \\
\hline $\mathrm{EF}, \%$ & 0.556 & 0.980 & 0.916 & 1.048 \\
\hline LVDd, mm & 0.203 & 1.049 & 0.975 & 1.128 \\
\hline LVDs, mm & 0.588 & 1.032 & 0.921 & 1.156 \\
\hline \multicolumn{5}{|l|}{ NYHA } \\
\hline II, III/IV & 0.263 & 1.969 & 0.601 & 6.458 \\
\hline \multicolumn{5}{|l|}{ Etiology } \\
\hline ICM & 0.077 & 2.931 & 0.891 & 9.645 \\
\hline DCM & 0.860 & 0.898 & 0.274 & 2.945 \\
\hline Valve & 0.319 & 0.351 & 0.045 & 2.748 \\
\hline \multicolumn{5}{|l|}{ Medication } \\
\hline ACEI/ARB & 0.542 & 0.662 & 0.175 & 2.495 \\
\hline Digitalis & 0.844 & 0.857 & 0.185 & 3.971 \\
\hline Statin & 0.632 & 1.337 & 0.408 & 4.383 \\
\hline
\end{tabular}

Table III. Multivariate Predictors of Recurrent Hospitalization for Worsening

\begin{tabular}{lcccc}
\hline & $P$ & Hazard ratio & \multicolumn{2}{c}{$95 \%$ CI } \\
\hline CD4/CD8 & 0.002 & 2.770 & 1.471 & 5.214 \\
Tregs, \% & 0.017 & 0.441 & 0.225 & 0.862 \\
\hline
\end{tabular}

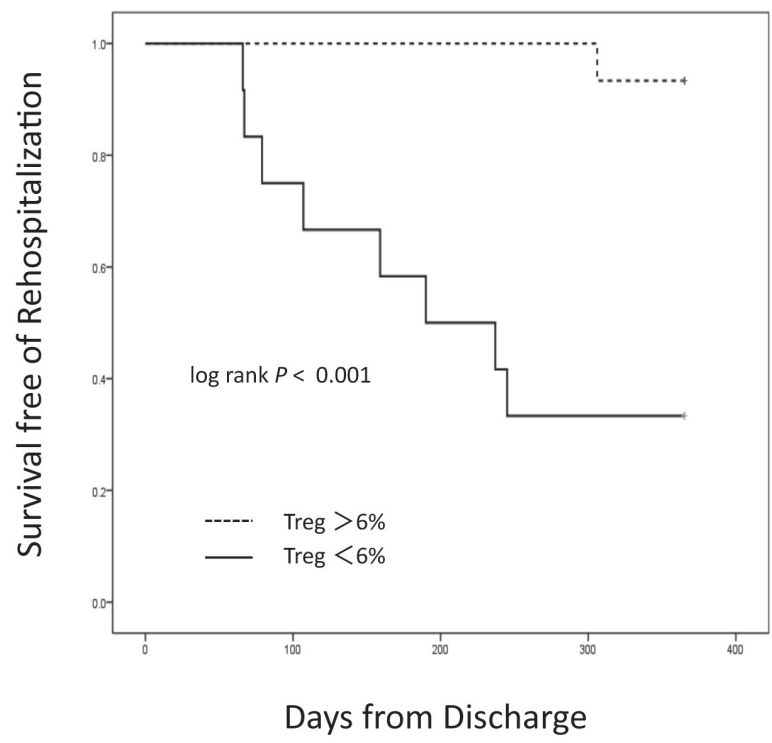

Figure 3. Kaplan-Meier curves demonstrating recurrent hospitalization free rates of $\mathrm{HF}$ patients during 12 months from discharge, expressed as a percentage of Tregs. Log-rank test, $P<0.001$. 
phocyte antigen 4 (CTLA-4), which only occurs after activation in effector T cells. CTLA-4 on Tregs binds to CD80/86 on antigen presenting cells (APCs) and transmits an inhibitory signal to effector T cells. ${ }^{15)}$ CTLA-4 expression on Tregs is critical for their suppression, which is mediated by CTLA-4dependent down-regulation of CD80/86 on APCs. Additionally, the HLADR expressing Tregs were reported to inhibit T cell proliferation and cytokine production via an early contactdependent mechanism associated with induction of Foxp3. Furthermore, Baecher-Allan, et al suggested that expression of HLADR on Tregs may be involved in homeostatic maintenance of Tregs in vivo by presenting self-antigens to other Tregs. ${ }^{16)}$ In our study, circulating $\mathrm{T}$ cells were markedly activated, which were shown as $\mathrm{CD}^{+} \mathrm{HLADR}^{+} \mathrm{T}$ cells in HF-REF patients. By contrast, HLADR ${ }^{+}$Tregs were significantly decreased in HF-REF patients, especially unstable patients. Our and previous findings suggest that $\mathrm{T}$ cells, which play a central role in the adaptive immunity, were identified as a critical cellular source for the persistent inflammation in $\mathrm{HF}^{6}$ )

The mechanism underlying the decrease in circulating Tregs in HF-REF patients remains unknown, although it may involve a change of differentiation of naive T cells. Th17 cells were originally identified as a new subset of $\mathrm{CD} 4^{+} \mathrm{T}$ cells that produce the inflammatory cytokine IL-17. Th17 cell-induced immune responses were also reported to play important roles in the pathogenesis of several inflammatory diseases and autoimmune disorders. ${ }^{17)}$ The Tregs are differentiated from naive $\mathrm{T}$ cells via TGF- $\beta$-induced Foxp3 expression. Interestingly, Th17 cells are also differentiated from naive T cells, and their generation is promoted by a combination of IL- 6 and TGF- $\beta$. Recent studies have demonstrated that IL- 6 has a very important role in regulating the balance between IL-17-producing Th17 cells and Tregs. In the presence of IL-6, TGF- $\beta$-induced Foxp3 expression is suppressed. ${ }^{18,19)}$ In our study, IL-6 expression was higher in HF-REF patients than in control subjects, suggesting that the differentiation of naive $\mathrm{T}$ cells may be switched from Tregs to Th17 cells, resulting in the decrease in the number of Tregs.

There is increasing evidence that Tregs control the development of atherosclerosis, a chronic inflammation disease. Antibody depletion of Tregs was reported to significantly increase atherosclerotic plaque size in mice. ${ }^{20)}$ In this study, some of the control subjects had arteriosclerosis such as angina pectoris and arteriosclerotic kidney, and $84 \%$ of control patients had risk factors for atherosclerosis including hypertension, diabetes mellitus, and dyslipidemia. Nevertheless, the frequency of Tregs in control subjects was significantly higher than that in HF-REF patients.

Finally, in this study, we demonstrated for the first time that circulating Tregs are an independent predictor for recurrent hospitalization for worsening HF. There are numerous reports that NT-proBNP and hs-CRP are predictors of poor outcome. ${ }^{21,22)}$ In this study, a decrease in the frequency of Tregs was a significant predictor of recurrent hospitalization, independent of other clinical and laboratory variables. We believe the decrease in Tregs is associated with inflammation, myocardial injury, and development of acute cardiovascular events in HF-REF patients. However, a direct causal relation between a decrease in Tregs and inflammation was not found in the study. Taken together, our results suggest that Tregs may be a useful predictor of recurrent hospitalization in 'unstable' HF patients.
There are several limitations in this study. We examined only peripheral circulating T-cells. To fully assess the role of Tregs on cardiac inflammation and left ventricular remodelling, future studies are required to examine the heart specific activation of Tregs, and develop interventions to increase the frequency of Tregs. Various medications, including renin-angiotensin-aldosterone system (RAAS) blockers and beta-blockers, have been shown to exhibit beneficial effects for treatment of HF. For example, the RAAS blockers ACEI and ARB were reported to be very effective at reducing HF-associated mortality. ${ }^{20,23)}$ Furthermore, beta-blockers such as carvedilol were reported to reduce mortality attributable to both worsening HF and sudden death, and inhibit progressive left ventricular remodelling and increased $\mathrm{EF}^{24,25)}$ In this study, most HF patients were receiving various medications such as beta-blockers and RAAS blockers. Thus, we were unable to determine an effect of medications on immunity.

In summary, we demonstrated that circulating Tregs are decreased in HF-REF patients hospitalized for worsening HF and are associated with inflammation and left ventricular dysfunction. Tregs may play a crucial role in controlling inflammation via suppression of cellular immune responses during the development of HF. Furthermore, circulating Tregs were an independent predictor of recurrent hospitalization for worsening $\mathrm{HF}$.

\section{ACKNOWLEDGMENTS}

The authors thank Ms. Ayami Yamagami for her excellent technical assistance

\section{REFERENCES}

1. Heeschen C, Hamm CW, Mitrovic V, Lantelme NH, White HD; Platelet Receptor Inhibition in Ischemic Syndrome Management (PRISM) Investigators. N-terminal pro-B-type natriuretic peptide levels for dynamic risk stratification of patients with acute coronary syndromes. Circulation 2004; 110: 3206-12.

2. Ho KK, Anderson KM, Kannel WB, Grossman W, Levy D. Survival after the onset of congestive heart failure in Framingham Heart Study subject. Circulation 1993; 88: 107-15.

3. Fonarow GC, Peacock WF, Phillips CO, Givertz MM, Lopatin M; ADHERE Scientific Advisory Committee and Investigators. Admission B-type natriuretic peptide levels and in-hospital mortality in acute decompensated heart failure. J Am Coll Cardiol 2007; 49: 1943-50.

4. Torre-Amione G. Immune activation in chronic heart failure. Am J Cardiol 2005; 95: 3C- 8C. (Review)

5. Vaduganathan M, Greene SJ, Gheorghiade M, et al. The immunological axis in heart failure: importance of the leukocyte differential. Heart Fail Rev 2013; 18: 835-45.

6. Yndestad A, Holm AM, Müller F, et al. Enhanced expression of inflammatory cytokines and activation markers in T-cells from patients with chronic heart failure. Cardiovasc Res 2003; 60: 141-6.

7. Sakaguchi S, Sakaguchi N, Asano M, Itoh M, Toda M. Immunologic self-tolerance maintained by activated $\mathrm{T}$ cells expressing IL-2 receptor alpha-chains (CD25). Breakdown of a single mechanism of self-tolerance causes various autoimmune disease. J Immunol 1995; 155: 1151-64.

8. Ochs HD, Ziegler SF, Torgerson TR. FOXP3 acts as a rheostat of immune response. Immunol Rev 2005; 203: 156-64. (Review)

9. Balandina A, Lécart S, Dartevelle P, Saoudi A, Berrih-Aknin S. Functional defect of regulatory $\mathrm{CD} 4^{+} \mathrm{CD} 25^{+} \mathrm{T}$ cells in the thymus 
of patients with autoimmune myasthenia gravis. Blood 2005; 105: 735-41.

10. Boissier MC, Assier E, Biton J, Denys A, Falgarone G, Bessis N. Regulatory T cells (Treg) in rheumatoid arthritis. Joint Bone Spine 2009; 76: 10-4. (Review)

11. Schneider-Hohendorf T, Stenner MP, Wiendl H, et al. Regulatory $\mathrm{T}$ cells exhibit enhanced migratory characteristics, a feature impaired in patients with multiple sclerosis. Eur J Immunol 2010; 40: 3581-90.

12. Tang TT, Ding YJ, Liao YH, et al. Defective circulating $\mathrm{CD} 4 \mathrm{CD} 25^{+} \mathrm{Foxp}^{+} \mathrm{CD} 127^{\text {low }}$ regulatory T-cells in patients with chronic heart failure. Cell Physiol Biochem 2010; 25: 451-8.

13. Kvakan H, Kleinewietfeld M, Qadri F, et al. Regulatory T cells ameliorate angiotensin II-induced cardiac damage. Circulation 2009; 119: 2904-12.

14. Matsumoto K, Ogawa M, Suzuki J, Hirata Y, Nagai R, Isobe M. Regulatory $\mathrm{T}$ lymphocytes attenuate myocardial infarction-induced ventricular remodeling in mice. Int Heart J 2011; 52: 382-7.

15. Wing K, Onishi Y, Prieto-Martin P, et al. CTLA-4 control over Foxp $3^{+}$regulatory T cell function. Science 2008; 322: 271-5.

16. Baecher-Allan C, Wolf E, Hafler DA. MHC class II expression identifies functionally distinct human regulatory T cells. J Immunol 2006; 176: 4622-31.

17. Wynn TA. $T(H)-17$ : a giant step from $T(H) 1$ and $T(H) 2$. Nature Immunol 2005; 6: 1069-70.

18. Chen W, Jin W, Hardegen N, et al. Conversion of peripheral $\mathrm{CD} 4^{+} \mathrm{CD} 25^{-}$naive $\mathrm{T}$ cells to $\mathrm{CD} 4^{+} \mathrm{CD} 25^{+}$regulatory $\mathrm{T}$ cells by
TGF-beta induction of transcription factor Foxp3. J Exp Med 2003; 198: 1875-86.

19. Bettelli E, Carrier Y, Gao W, et al. Reciprocal development pathways for the generation of pathogenic effector TH17 and regulatory T cells. Nature 2006; 441: 235-8.

20. Xie JJ, Wang J, Tang TT, et al. The Th17/Treg functional imbalance during atherogenesis in ApoE(-/-) mice. Cytokine 2010; 49: 185-93.

21. Dargie HJ. Effect of carvedilol on outcome after myocardial infarction in patients with left-ventricular dysfunction: the CAPRICORN randomised trial. Lancet 2001; 357: 1385-90.

22. Hori M, Sasayama S, Kitabatake A, et al; MUCHA Investigators. Low-dose carvedilol improves left ventricular function and reduces cardiovascular hospitalization in Japanese patients with chronic heart failure: the Multicenter Carvedilol Heart Failure Dose Assessment (MUCHA) trial. Am Heart J 2004; 147: 324-30.

23. Januzzi JL, van Kimmenade R, Lainchbury J, et al. NT-proBNP testing for diagnosis and short-term prognosis in acute destabilized heart failure: an international pooled analysis of 1256 patients: the International Collaborative of NT-proBNP Study. Eur Heart J 2006; 27: 330-7.

24. Anand IS, Latini R, Florea VG, et al; Val-HeFT Investigators. Creactive protein in heart failure: prognostic value and the effect of valsartan. Circulation 2005; 112: 1428-34.

25. The SOLVD Investigators. Effect of enalapril on survival in patients with reduced left ventricular ejection fractions and congestive heart failure. N Engl J Med 1991; 325: 293-302. 